\title{
Projective and free matricially normed spaces
}

\author{
A. Ya. Helemskii
}

\begin{abstract}
We study metrically projective and metrically free matricially normed spaces. We describe these spaces in terms of a special space $\widehat{M}_{n}$, the space of $n \times n$ matrices, endowed with a special matrix-norm. We show that metrically free matricially normed spaces are matricial $\ell_{1}$-sums of some distinguished families of matricially normed spaces $\widehat{M}_{n}$, whereas metrically projective matricially normed spaces are complete direct summands of matricial $\ell_{1}$-sums of arbitrary families of the spaces $\widehat{M}_{n}$. At the end we specify the underlying normed space of $\widehat{M}_{n}$ and show that the spaces $\widehat{M}_{n} ; n>1$ do not belong to any of the classes $L^{p} ; p \in[1, \infty]$, introduced by Effros and Ruan. However, in a certain sense the behavior of $\widehat{M}_{n}$ resembles that of $L^{1}$-spaces.
\end{abstract}

\section{Introduction}

The notion of a matricially normed space was introduced by Effros and Ruan [3]. Soon, after the discovery of Ruan representation theorem [15], the most attention was concentrated to the outstanding special class of these structures, namely the $L^{\infty}$-spaces, more often now called (abstract) operator spaces. (see the textbooks [4, 12, 14, 2]). However, already in [3, 15. it was demonstrated that matricially normed spaces are a subject of considerable interest also outside the class of operator spaces. In particular, according to these papers, one can successfully study the Haagerup tensor

\footnotetext{
Keywords: matricially normed spaces, metrically projective spaces, metrically free spaces, spaces $\widehat{M}_{n}$.

Mathematics Subject Classification (2010): 46L07, 46M05.

This research was supported by the Russian Foundation for Basic Research (grant No. 15-01-08392).
} 
product of these spaces. Later it was shown in [10] that another important tensor product of operator spaces, the projective tensor product, also can be studied in the context of general matricially normed spaces.

In this paper we introduce and study the closely connected notions of a metrically projective and a metrically free matricially normed space. In the realm of operator spaces the definition of a metrically projective space resembles what was called by Blecher [1] (just) projective space, but differs from the latter. In the classical context of Banach spaces the metric projectivity appeared, under a different name, in the old paper of Graven [5].

We first characterize metrically free matricially normed spaces. Then, using the well known general categorical connection between freeness and projectivity, we characterize metrically projective matricially normed spaces. We describe the spaces in both classes in terms of the special space $\widehat{M}_{n}$, the space of $n \times n$-matrices endowed with a special matrix-norm. We show that metrically free spaces are matricial $\ell_{1}$-sums of some specified families of spaces $\widehat{M}_{n}$, whereas metrically projective spaces are complete direct summands of matricial $\ell_{1}$-sums of arbitrary families of spaces $\widehat{M}_{n}$.

The latter result, concerning projectivity, resembles Blecher's Theorem 3.10 in [1], and in fact we were inspired by that. Note that the mentioned theorem, extended in a straightforward way to general matricially normed spaces, also can be deduced from the description of metrically free matricially normed spaces, however after some elaboration of our general categorical tools; cf. [8]. But we leave this material outside the scope of the present paper.

The contents of the paper are as follows.

Section 2 contains some preliminary definitions.

In Section 3 we introduce our main matricially normed space $\widehat{M}_{n}$.

In Section 4 we prepare our tools from category theory. We consider the so-called rigged categories (well known under many different names), define projective objects in such a category and show that the metric projectivity of matricially normed spaces is a particular case of this general categorical projectivity. Then we introduce, within the frame-work of a rigged category, the notion of a free object. We recall several general categorical observations that will be used in later sections, notably the characterization of projective objects as retracts of free objects.

In Section 5 we fix $n \in \mathbb{N}$ and introduce the special rig ' $\odot_{n}$ ', playing, in a sense, the role of a 'building brick' for the rig, responsible for the 
metric projectivity. We show that the $\odot_{n}$-free object with a one-point base is exactly the matricially normed space $\widehat{M}_{n}$. The proof heavily relies on properties of a distinguished $n^{2} \times n^{2}$-matrix $\mathbb{I}$. The latter, in the guise of an element of $M_{n} \otimes M_{n}$, is $\sum_{i j} e_{j i} \otimes e_{i j}$, where $e_{i j}$ denotes the elementary matrix with 1 on the $i j$-th place.

In Section 6 we apply the results of the previous sections to obtain our main results; namely, the above-mentioned description of metrically free and (as a corollary) of metrically projective matricially normed spaces.

Finally, in Section 7 we obtain some (far from complete) information about the structure of the space $\widehat{M}_{n}$. First, we find its underlying normed space: it turns out to be the space of $n \times n$-matrices with the trace class norm. Thus, it is the same as the underlying space of the operator space $T_{n}$, playing the main role in the description of projective operator spaces in [1]. However, as a matricially normed space, $\widehat{M}_{n}$ is profoundly different from $T_{n}$. We show that it does not belong to any of the classes $L^{p} ; 1<p \leq \infty$ of Effros/Ruan, which is not surprising, and (what is somehow surprising to the author) to the class $L^{1}$ as well. Nevertheless, in a certain sense the behavior of $\widehat{M}_{n}$ resembles that of $L^{1}$-spaces.

\section{Initial definitions}

In what follows, we denote the space of $n \times n$-matrices, as a pure algebraic object, by $M_{n}$, and the same space, endowed by the operator norm $\|\cdot\|_{o}$ or the trace norm $\|\cdot\|_{t}$, by $\mathbb{M}_{n}$ and $\mathbb{T}_{n}$, respectively. If $E$ is a normed space, we denote by $B_{E}$ its closed unit ball. The identity operator on a linear space $E$ is denoted by $\mathbf{1}_{E}$.

Let $E$ be a linear space, $M_{n}(E)$ the space of $n \times n$-matrices with entries from $E$. We identify $M_{n}(E)$ with the tensor product $M_{n} \otimes E$. According to our convenience, we shall use either 'matrix guise' or 'tensor guise' of this space. We denote the $E$-valued diagonal block-matrix with matrices $u_{1}, \ldots, u_{k}$ on the diagonal by $u_{1} \oplus \ldots \oplus u_{k}$.

Definition 1. (Effros/Ruan [4]) A sequence of norms $\|\cdot\|_{n}$ on $M_{n}(E)$ : $n \in \mathbb{N}$ is called a matrix-norm on $E$, if it satisfies the two following conditions:

Axiom 1. For $u \in M_{n}(E)$ and $n, m \in \mathbb{N}$ we have $\|u \oplus 0\|_{n+m}=\|u\|_{n}$. Here 0 is the zero matrix in $M_{m}(E)$.

Axiom 2. For $u \in M_{n}(E)$ and $S \in M_{n}$ we have $\|S u\|_{n} \leq\|S\|_{o}\|u\|_{n}$ and $\|u S\|_{n} \leq\|u\|_{n}\|S\|_{o}$. 
A space $E$, endowed with a matrix-norm, is called matricially normed space. The normed space, identified with $M_{1}(E)$, is called underlying normed space of our matricially normed space.

Two examples that we shall need are the matricially normed spaces $\mathbb{C}_{\text {min }}$ and $\mathbb{C}_{\max }$. The first one is $\mathbb{C}$ with the matrix-norm, arising after the identifying, for every $m \in \mathbb{N}$, of $M_{m}(\mathbb{C})$ with $\mathbb{M}_{m}$, whereas the second one is $\mathbb{C}$ with the matrix-norm, arising after the identifying of $M_{m}(\mathbb{C})$ with $\mathbb{T}_{m}$.

Every subspace $F$ of a matricially normed space is, of course, a matricially normed space with respect to induced norms in $M_{n}(F) \subseteq M_{n}(E)$ for every $n$. We call it matricially normed subspace of $E$.

Now let $E$ and $F$ be linear spaces, $\varphi: E \rightarrow F$ a linear operator. The operator $\varphi_{n}: M_{n}(E) \rightarrow M_{n}(F):\left(x_{i j}\right) \mapsto\left(\varphi\left(x_{i j}\right)\right)$, is called $n$-th amplification of $\varphi$. (In the 'tensor approach' $\varphi_{n}$ is, of course, $\mathbf{1}_{M_{n}} \otimes \varphi$ ).

If our $E$ and $F$ are matricially normed spaces, then we call $\varphi$ completely bounded, if $\sup \left\{\left\|\varphi_{n}\right\| ; n \in \mathbb{N}\right\}<\infty$. We denote this supremum by $\|\varphi\|_{c b}$.

If, in the previous context, every amplification is a contractive operator (that is $\|\varphi\|_{c b} \leq 1$ ), we say that $\varphi$ is completely contractive. (This is the most important class of operators in the present paper). The set of completely contractive operators between $E$ and $F$ is denoted by $\mathcal{C C}(E, F)$. If every amplification is isometric, strictly coisometric or isometric isomorphism, we say that $\varphi$ is completely isometric, completely strictly coisometric or completely isometric isomorphism, respectively. Here we recall that the operator between normed spaces $E$ and $F$ is called strictly coisometric (or exact quotient map), if it maps $B_{E}$ onto $B_{F}$.

The (non-additive) category with matricially normed spaces as objects and completely contractive operators as morphisms is denoted by $\mathbf{M N}_{\mathbf{1}}$. Evidently, isomorphisms in this category are completely isometric isomorphisms, defined above.

Definition 2. A matricially normed space $P$ is called metrically projective, if, for every completely strictly coisometric operator $\tau$ between matricially normed spaces, say $E$ and $F$, and every completely contractive operator $\varphi: P \rightarrow F$ there exists a completely contractive operator $\psi: P \rightarrow E$, making the diagram

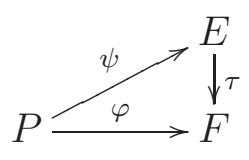

commutative. 


\section{Construction of the matricially normed spaces $\widehat{M}_{n}$}

From now on and until we state otherwise, we fix some $n \in \mathbb{N}$. Sometimes, if there is no danger of confusion, we omit index $n$.

We begin with pure algebraic preparations.

Suppose we are given a linear space $E$. Let us introduce the operator

$$
\iota^{E}: M_{n}(E) \rightarrow \mathcal{L}\left(M_{n}, E\right)
$$

where $\mathcal{L}(\cdot, \cdot)$ is the symbol of the space of linear operators. It takes an $n \times n$-matrix $\left(x_{i j}\right)$ with entries in $E$ to the operator $\left(\lambda_{i j}\right) \mapsto \sum_{i j} \lambda_{j i} x_{i j} \in E$, where $\left(\lambda_{i j}\right) \in M_{n}$. Equivalently, if we use the 'tensor guise' of $M_{n}(E)$, then $\iota^{E}: M_{n} \otimes E \rightarrow \mathcal{L}\left(M_{n}, E\right)$ is well defined by taking an elementary tensor $a \otimes x$ to the operator $b \mapsto \operatorname{tr}(a b) x$. Here and onwards $\operatorname{tr}$ denotes the trace of a matrix. Obviously, $\iota^{E}$ is a linear isomorphism.

It is convenient to denote, for a given $v \in M_{n}(E)$, the operator $\iota^{E}(v)$ by $\varphi^{v}: M_{n} \rightarrow E$.

Now consider all possible couples $(E, v)$, where $E$ is a matricially normed space and $v \in B_{M_{n}(E)}$. In what follows, we refer them as proper couples.

Definition 3. For every $m \in \mathbb{N}$ and $u \in M_{m}\left(M_{n}\right)$ we set

$$
\|u\|_{m}:=\sup \left\{\left\|\left(\varphi^{v}\right)_{m}(u)\right\|_{m}\right\}
$$

where supremum is taken over all proper couples.

(We recall that $\left(\varphi^{v}\right)_{m}: M_{m}\left(M_{n}\right) \rightarrow M_{m}(E)$ takes an $m \times m-$ matrix $\left(a^{k l}\right)$ with entries in $M_{n}$ to the $m \times m$-matrix $\left(\varphi^{v}\left(a^{k l}\right)\right)$ with entries in $E$.

Proposition 1. The indicated supremum is finite. Moreover, $\|u\|_{m}$ does not exceed the sum of modules of matrix entries after the identification of $M_{m}\left(M_{n}\right)$ with $M_{m n}$.

Proof. Take $u \in M_{m}\left(M_{n}\right) ; u=\left(a^{k l}\right), a^{k l}=\left(\lambda_{i j}^{k l}\right) \in M_{n}$. We must show that for every proper couple $(E, v)$ we have $\left\|\left(\varphi^{v}\right)_{m}(u)\right\| \leq \sum_{k l} \sum_{i j}\left|\lambda_{i j}^{k l}\right|$.

If $v=\left(x_{i j}\right) ; x_{i j} \in E$, then, since $v \in B_{M_{n}(E)}$, and $E$ is a matricially normed space, we have $\left\|x_{i j}\right\| \leq 1$ for all $i, j$. Hence $\left\|\varphi^{v}\left(a^{k l}\right)\right\| \leq \sum_{i j}\left|\lambda_{i j}^{k l}\right|$. On the other hand, using again that $E$ is a matricially normed space, we have $\left\|\left(v a^{v}\right)_{m}(u)\right\| \leq \sum_{k l}\left\|\varphi^{v}\left(a^{k l}\right)\right\|$.

Theorem 1. The sequence of functions $u \mapsto\|u\|_{m} ; m \in \mathbb{N}$ is a matrixnorm on $M_{n}$. 
Proof. First, we show that the function $u \mapsto\|u\|_{m}$ is a seminorm on $M_{m}\left(M_{n}\right)$ and then we check the Axioms 1 and 2. All three assertions are proved by a similar argument. Namely, we use the respective properties of matricially normed spaces $E$ in proper couples and then the definition of $\|u\|_{m}$ as the relevant supremum. For example, the first estimation in Axiom 2 follows from the relations

$$
\left\|\left(\varphi^{v}\right)_{m}(S u)\right\|=\left\|S\left(\varphi^{v}\right)_{m}(u)\right\| \leq\|S\|_{o}\left\|\left(\varphi^{v}\right)_{m}(u)\right\| \leq\|S\|_{o}\|\| u \|_{m} .
$$

Finally, we prove that our seminorm is actually a norm. Take $u \in$ $M_{m}\left(M_{n}\right) ; u=\left(a^{k l}\right) ; a^{k l}=\left(\lambda_{i j}^{k l}\right) \in M_{n}$. If $u \neq 0$, then $\lambda_{j^{\prime} i^{\prime}}^{k^{\prime} l^{\prime}} \neq 0$ for a certain $k^{\prime}, l^{\prime}$ and $i^{\prime}, j^{\prime}$. Choose a proper couple with $v=\left(x_{i j}\right) \in M_{n}(E)$ such that $x_{i j} \neq 0$ if, and only if $i=i^{\prime}$ and $j=j^{\prime}$, and also $\left\|x_{i^{\prime} j^{\prime}}\right\| \leq 1$. Then we see that $\left(\varphi^{v}\right)_{m}$ takes $u$ to the matrix $\left(y_{k l}\right) \in M_{m}(E)$ with $y_{k^{\prime} l^{\prime}} \neq 0$. Therefore $\left\|\left(\varphi^{v}\right)_{m}(u)\right\|>0$, hence $\|u\|>0$.

We denote the resulting matricially normed space by $\widehat{M}_{n}$.

In particular, it is easy to show that $\widehat{M}_{1}$ is just $\mathbb{C}_{\max }$. The structure of $\widehat{M}_{n}$ for bigger $n$ is not so transparent; we shall see this in our last section.

\section{Projectivity and freeness in rigged categories}

Definition 4. Let $\mathcal{K}$ be an arbitrary category. A rig of $\mathcal{K}$ is a faithful covariant functor $\square: \mathcal{K} \rightarrow \mathcal{L}$, where $\mathcal{L}$ is another category. A pair $(\mathcal{K}, \square)$, consisting of a category and its rig, is called rigged category.

We call a morphism $\tau$ in $\mathcal{K}$ admissible, if $\square(\tau)$ is a retraction in $\mathcal{L}$.

Definition 5. An object $P$ in $\mathcal{K}$ is called $\square$-projective, if, for every admissible morphism $\tau: Y \rightarrow X$ and every morphism $\varphi: P \rightarrow X$, there exists a morphism $\psi: P \rightarrow Y$, making the diagram (D1) commutative.

Our principal example. Consider the covariant functor

$$
\odot: \mathrm{MN}_{1} \rightarrow \text { Set }
$$

taking a matricially normed space $E$ to the cartesian product $\mathrm{X}_{m=1}^{\infty} B_{M_{m}(E)}$. Thus, the elements of the set $\odot(E)$ are sequences $\left(w_{1}, \ldots, w_{m}, \ldots\right)$ where $w_{m} \in B_{M_{m}(E)}$. As to the action of our functor on morphisms, it takes a completely contractive operator $\varphi: E \rightarrow F$ to the map

$$
\odot(\varphi): \odot(E) \rightarrow \odot(F),\left(w_{1}, \ldots, w_{m}, \ldots\right) \mapsto\left(\varphi\left(w_{1}\right), \ldots, \varphi\left(w_{m}\right), \ldots\right) .
$$

It is clear that we have obtained a rigged category. Note the following obvious statement. 
Proposition 2. (i) A morphism in $\mathbf{M N}_{\mathbf{1}}$ is $\odot$-admissible if, and only if it is a completely strictly coisometric operator.

(ii) $\odot$-projective objects in $\mathbf{M N}_{\mathbf{1}}$ are exactly metrically projective matricially normed spaces.

Return to general rigged categories. The following concept is well known under different names.

Definition 6. Let $M$ be an object in $\mathcal{L}$. An object $\operatorname{Fr}(M)$ in $\mathcal{K}$ is called $\square$-free object with the base $M$, if for every $X \in \mathcal{K}$, there exists a bijection

$$
\mathcal{I}_{X}: \mathbf{h}_{\mathcal{L}}(M, \square X) \rightarrow \mathbf{h}_{\mathcal{K}}(\operatorname{Fr}(M), X)
$$

between the respective sets of morphisms, natural on $X$. We say that a rigged category admits freeness, if every object in $\mathcal{L}$ is a base of a free object in $\mathcal{K}$.

Remark. According to [11, Chs. III,IV], to say that a rigged category admits freeness is equivalent to say that $\square$ has a left adjoint functor.

The following observations show the practical use of the freeness. They are actually well known and can be extricated, as particular cases or easy corollaries, from some general facts, contained in [11, Chs. III,IV].

Proposition 3. Suppose that our rigged category admits freeness. Then

(i) every object in $\mathcal{K}$ is the range of an admissible morphism with a free domain.

(ii) an object in $\mathcal{K}$ is is projective if, and only if it is a retract of a free object. In particular, all free objects in $\mathcal{K}$ are projective.

It was proved in [8] that the rig, obtained from $\odot$ by the restriction of $\mathbf{M N}_{1}$ to its subcategory of operator spaces, admits freeness, and its free objects are the so-called $\oplus_{1}$-sums of certain families of 'building bricks'. The latter are the spaces, considered, as operator spaces, as dual to the 'concrete' operator space $\mathbb{M}_{n}$. (These spaces were already used in [1]). A similar result was obtained in [9] in more general context of operator modules over operator algebras.

But our aim is to find free objects in the 'whole' rigged category $\left(\mathbf{M N}_{1}, \odot\right)$. To begin with, we shall find free objects in another rigged category that is, speaking informally, a 'small part' of $\left(\mathbf{M N}_{\mathbf{1}}, \odot\right)$. 


\section{The rig $\odot_{n}$ and its free objects with the one-point base}

With $n$ still fixed, we consider the covariant functor

$$
\odot_{n}: \mathrm{MN}_{1} \rightarrow \text { Set }
$$

taking a matricially normed space $E$ to the set $B_{M_{n}(E)}$ and taking a completely contractive operator to its restriction to the respective unit balls. Evidently, we get a rig.

Now we need some preparation. First, recall the operator $\iota^{E}$ (see $\left.(1)\right)$.

Proposition 4.Let $E, F$ be linear spaces, $\psi: E \rightarrow F$ an operator. Then the diagram

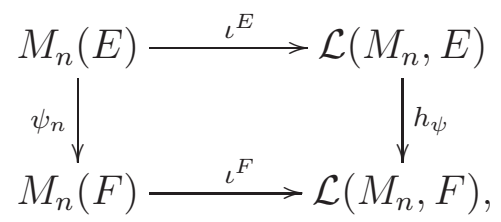

where $h_{\psi}$ takes an operator $\chi$ to the composition $\psi \chi$, is commutative.

Proof. A convenient way to check this is to use the 'tensor guise' of $M_{n}(E)$ and look at elementary tensors in $M_{n} \otimes E$.

Recall the matrices $e_{i j} \in M_{n}$ and $\mathbb{I} \in M_{n}\left(M_{n}\right)$ from Section 1. A routine calculation gives

Proposition 5. The operator $\varphi^{\mathbb{I}}$ is just $\mathbf{1}_{M_{n}}$.

Proposition 6. For every linear space $E$ and $v \in M_{n}(E)$ we have $\left(\varphi^{v}\right)_{n}(\mathbb{I})=v$.

Proof. Represent $v$ as $\sum e_{i j} \otimes x_{i j} ; x_{i j} \in E$. Then we have $\varphi^{v}\left(e_{i j}\right)=x_{j i}$, hence $\left(\varphi^{v}\right)_{n}(\mathbb{I})=\sum_{i j} e_{j i} \otimes \varphi^{v}\left(e_{i j}\right)=\sum_{i j} e_{j i} \otimes x_{j i}=v$.

This, together with (2), implies

Proposition 7. The norm of $\mathbb{I}$ in $M_{n}\left(\widehat{M}_{n}\right)$ is 1 .

Theorem 2. The matricially normed space $\widehat{M}_{n}$ is $\odot_{n}$-free with a onepoint set as its base.

Proof. Let $E$ be a matricially normed space, and $\{\star\}$ a one-point set. According to (4), we must construct a bijection

$$
\mathcal{I}_{E}^{n}: \mathbf{h}_{\mathbf{S e t}}\left(\{\star\}, \odot_{n}(E)\right) \rightarrow \mathbf{h}_{\mathbf{M N}_{\mathbf{1}}}\left(\widehat{M}_{n}, E\right),
$$

or, equivalently, a bijection $\mathcal{I}_{E}^{n}: B_{M_{n}(E)} \rightarrow \mathcal{C C}\left(\widehat{M}_{n}, E\right)$, natural on $E$. Take $v \in B_{M_{n}(E)}$ and consider $\varphi^{v}$ as an operator between the matricially normed 
spaces $\widehat{M}_{n}$ and $E$. Then for every $m$ and $u \in M_{m}\left(\widehat{M}_{n}\right)$ we have, by $(2)$, that $\|u\|_{m} \geq\left\|\left(\varphi^{v}\right)_{m}(u)\right\|$. This means that $\varphi^{v}: \widehat{M}_{n} \rightarrow E$ is completely contractive. Thus, the map $\iota^{E}$ has the well defined restriction to $B_{M_{n}(E)}$ and $\mathcal{C C}\left(\widehat{M}_{n}, E\right)$. It is this restriction that we choose as $\mathcal{I}_{E}^{n}$. Show that it has required properties.

The commutative diagram (D2), being restricted to the respective unit balls and sets $\mathcal{C C}(\cdot, \cdot)$, demonstrates that our constructed $\mathcal{I}_{E}^{n}$ is natural on $E$. Also $\mathcal{I}_{E}^{n}$ is obviously injective. It remains to show that it is surjective.

Take an arbitrary $\psi: \mathcal{C C}\left(\widehat{M}_{n}, E\right)$ and consider the diagram

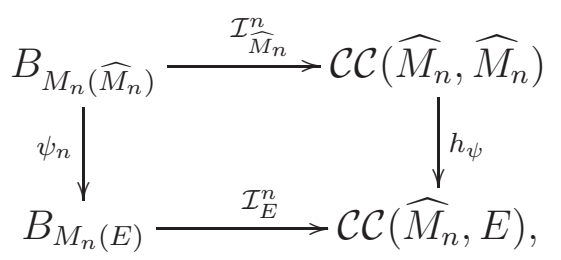

the relevant restriction of the diagram (D2) after choosing $\widehat{M}_{n}$ as $E$ and $E$ as $F$. Now recall that the element $\mathbb{I}$ belongs, by Proposition 7 , to $B_{M_{n}\left(\widehat{M}_{n}\right)}$, and Proposition 5 implies that $\mathcal{I}_{\widehat{M}_{n}}^{n}(\mathbb{I})=\mathbf{1}_{\widehat{M}_{n}}$. But $h_{\psi}\left(\mathbf{1}_{\widehat{M}_{n}}\right)=\psi$, and our diagram is commutative. Therefore $\psi=\mathcal{I}_{E}^{n}\left(\psi_{n}(\mathbb{I})\right)$, and we are done. $\square$.

\section{Characterization of free and projective spaces}

To move from the rig $\odot_{n}$ and its free objects with one-point bases to the 'whole' $\odot$ and its free objects with arbitrary bases, we need the following well known categorical concept (cf., e.g., [7, Ch.2] or [11]).

Let $X_{\nu} ; \nu \in \Lambda$ be a family of objects in an (arbitrary) category $\mathcal{K}$. We recall that a pair $\left(X,\left\{i_{\nu} ; \nu \in \Lambda\right\}\right)$, where $X$ is an object, and $i_{\nu}: X_{\nu} \rightarrow X$ are morphisms in $\mathcal{K}$, is said to be the coproduct of this family, if, for every object $Y$ and a family of morphisms $\psi_{\nu}: X_{\nu} \rightarrow Y$ there is a unique morphism $\psi: X \rightarrow Y$ such that we have $\psi i_{\nu}=\psi_{\nu}$ for every $\nu \in \Lambda$.

(We speak about 'the' coproduct because it is unique up to a categorical isomorphism, compatible with the respective coproduct injections.)

The mentioned $X$, denoted in a detailed form by $\coprod\left\{X_{\nu} ; \nu \in \Lambda\right\}$, is referred as the coproduct object, and $i_{\nu}$ 's as the coproduct injections. The morphism $\psi$ is called the coproduct of the morphisms $\psi_{\nu}$ and denoted by $\coprod\left\{\psi_{\nu} ; \nu \in \Lambda\right\}$.

We say that $\mathcal{K}$ admits coproducts, if every family of its objects has the coproduct. 
Of course, the category Set admits coproducts: the coproduct of a family of sets is their disjoint union, with obvious coproduct injections. Also it is well known that the category Nor $_{1}$ of normed spaces and contractive operators also admits coproducts: the coproduct of a family of normed spaces is their (classical) $\ell_{1}$-sum.

Now suppose we have a family $E_{\nu} ; \nu \in \Lambda$ of matricially normed spaces. Consider their algebraic sum $E:=\oplus\left\{E_{\nu} ; \nu \in \Lambda\right\}$ and identify, for every $m \in \mathbb{N}$, the linear spaces $M_{m}(E)$ and $\oplus\left\{M_{m}\left(E_{\nu}\right) ; \nu \in \Lambda\right\}$. Endow every $M_{m}(E)$ with the norm of the $\ell_{1}$-sum of normed spaces. Then we easily see that we made $E$ a matricially normed space. We call it matricial $\ell_{1}$-sum of a given family. As an easy corollary of the structure of coproducts in Nor , $_{1}$ we obtain

Proposition 8. The matricial $\ell_{1}$-sum of a given family of matricially normed spaces is the coproduct of this family in $\mathbf{M N}_{\mathbf{1}}$ with the natural embeddings $i_{\nu}: E_{\nu} \rightarrow E$ as the coproduct injections. Thus, the category $\mathbf{M N}_{1}$ admits coproducts.

Remark. The full subcategory of $\mathbf{M N}_{1}$, consisting of operator spaces, also admits coproducts, but the respective construction is necessarily more sophisticated. It was shown by Blecher [1, Sect. 3].

We turn to $\odot$-free objects that in what follows will be referred as metrically free matricially normed spaces. At first we concentrate on the case of the one-point base.

From now on we 'release' $n$. Denote by $\widehat{M}_{\infty}$ the matricial $\ell_{1}$-sum ( = coproduct in $\mathbf{M N}_{\mathbf{1}}$ ) of the family $\left\{\widehat{M}_{n} ; n \in \mathbb{N}\right\}$.

Theorem 3. The metrically free matricially normed space with a onepoint base, say $\{\star\}$, does exist, and it is $\widehat{M}_{\infty}$.

Proof. Let $E$ be an arbitrary matricially normed space. We must construct a bijection

$$
\mathcal{I}_{E}^{\infty}: \mathbf{h}_{\text {Set }}(\{\star\}, \odot(E)) \rightarrow \mathbf{h}_{\mathbf{M N}_{\mathbf{1}}}\left(\widehat{M}_{\infty}, E\right),
$$

natural on $E$. The first of the indicated sets can be identified with the set of sequences $\mathbf{w}=\left(w_{1}, \ldots, w_{n}, \ldots\right) ; w_{n} \in B_{M_{n}(E)}$.

By Theorem 2, for every $n$, after relevant identifications, there exists a bijection $\mathcal{I}_{E}^{n}: B_{M_{n}(E)} \rightarrow \mathcal{C C}\left(\widehat{M}_{n}, E\right)$, taking $w_{n}$ to the operator $\varphi^{w_{n}}$. Thus every sequence $\mathbf{w}$ gives rise to a family of completely contractive operators $\varphi^{w_{n}}: \widehat{M}_{n} \rightarrow E$. Denote by $\varphi^{\mathrm{w}}: \widehat{M}_{\infty} \rightarrow E$ the coproduct of these $\varphi^{w_{n}}$. 
Taking every $\mathbf{w}$ to $\varphi^{\mathbf{w}}$, we obtain, modulo the mentioned identifications, a map $\mathcal{I}_{E}^{\infty}$ between the sets, indicated in (6). Note that for all completely contractive operators $\psi: E \rightarrow F$, where $E, F$ are matricially normed spaces, we obviously have $\psi\left(\coprod\left\{\varphi^{v_{n}}\right\}\right)=\coprod\left\{\psi \varphi^{v_{n}}\right\}$. Therefore, knowing that every $\mathcal{I}_{E}^{n} ; n \in \mathbb{N}$ is natural on $E$, we obtain that $\mathcal{I}_{E}^{\infty}$ is natural on $E$. Finally, since every $\mathcal{I}_{E}^{n}$ is a bijection, $\mathcal{I}_{E}^{\infty}$ is also a bijection.

To pass from one-point sets, as bases of free objects, to arbitrary sets, we can use the following simple categorical observation. Let $\square: \mathcal{K} \rightarrow \mathcal{L}$ be an arbitrary rig.

Proposition 9. Suppose that we are given a family $F_{\nu} ; \nu \in \Lambda$ of free objects with bases $M_{\nu}$. Further, suppose that there exist the coproducts $F:=$ $\coprod\left\{F_{\nu} ; \nu \in \Lambda\right\}$ and $M:=\coprod\left\{M_{\nu} ; \nu \in \Lambda\right\}$ in $\mathcal{K}$ and $\mathcal{L}$, respectively. Then $F$ is a free object with the base $M$.

Proof. See, e.g., [8, Prop. 2.13].

Since every set $M$ is the coproduct of its one-point subsets, this proposition immediately implies

Theorem 4. For every set $M$, there exists a metrically free matricially normed space with the base $M$, and it is the matricial $\ell_{1}$-sum of the family of copies of the matricially normed space $\widehat{M}_{\infty}$, indexed by points of $M$. Thus, the rigged category $\left(\mathbf{M N}_{\mathbf{1}}, \odot\right)$ admits freeness.

Now we want to pass from free to projective matricially normed spaces. To make the formulation more geometrically transparent, we say that a matricially normed space $F$ is a a complete direct summand of a matricially normed space $E$, if $F$ is completely isometrically isomorphic to a matricially normed subspace $G$ of $E$, and there is a completely contractive projection of $E$ onto $G$. We have an obvious

Proposition 10. A matricially normed space $F$ is a retract in $\mathbf{M N}_{\mathbf{1}}$ of a matricially normed space $E$ if, and only if $F$ is a complete direct summand of $E$.

In what follows, we use a simple general-categorical observation, concerning an arbitrary rig.

Proposition 11. (i) A retract of $a \square$-projective object is $\square$-projective

(ii) the coproduct of a family of $\square$-projective objects (if, of course, it does exist) is $\square$-projective. 
We call a matricially normed space $\widehat{M}$-composed, if it is a matricial $\ell_{1}-$ sum of some family of spaces such that each of summands is $\widehat{M}_{n}$ for some $n \in \mathbb{N}$.

Theorem 5. (i) Every matricially normed space is an image of a completely strictly coisometric operator with the $\widehat{M}$-composed space as its domain

(ii) A matricially normed space is metrically projective if, and only if it is a complete direct summand of a $\widehat{M}$-composed space.

Proof. Combining Propositions 2(i) and 3(i) with Theorem 4 and Proposition 2, we obtain (i). Combining Propositions 3(ii) and 10 with Theorem 4, we obtain the 'only if' part of (ii). To prove the rest, we observe that every space $\widehat{M}_{n}$ is, of course, a complete direct summand of the space $\widehat{M}_{\infty}$, hence, by Propositions 10 and 11(i), combined with Theorem 3, it is metrically projective. It remains to use Propositions 8 and 11(ii), and then Propositions 3(ii) and (again) 10 .

Remark. Blecher [1] considered a different kind of projectivity. This was the operator space version of the 'lifting property' of some Banach spaces (cf., e.,g., [13, p. 133]), studied in the classical context by Grothendieck [6]. This kind of projectivity also can be treated within the general frame-work of a rigged category and its free objects, but after a kind of elaboration of our scheme. Such an approach was used for operator spaces, in [8, 9]. As to general matricially normed spaces, this approach leads to the following version of Theorem 3.10 in [1]:

A matricially normed space is projective (in the just mentioned sense) if, and only if it is almost a direct summand of a $\widehat{M}$-composed space.

The definition of an almost direct summand repeats word by word the Definition 3.8 in [1] that was given for operator spaces.

\section{Some properties of the matricially normed space $\widehat{M}_{n}$}

In this section we again fix a natural $n$.

Theorem 6. The underlying normed space of $\widehat{M}_{n}$ is $\mathbb{T}_{n}$.

Proof. Denote the norm on $M_{1}\left(\widehat{M}_{n}\right)$ by $\|\cdot\|_{\bullet}$. Take an arbitrary element, say $a$, in $M_{1}\left(\widehat{M}_{n}\right)$. First, we prove that $\|a\|_{\bullet} \leq\|\cdot\|_{t}$. Accordingly, our task is to show that for every proper couple $(E, v)$ we have $\left\|\varphi^{v}(a)\right\| \leq\|a\|_{t}$.

Consider the commutative diagram (D2) with $\mathbb{C}$ as $F$ and an arbitrary functional $f$ on $E$ as $\psi$. Fix $v \in M_{n}(E)$ and denote, for brevity, the matrix 
$f_{n}(v) \in M_{n}(\mathbb{C})$ by $b$. Then for our $a$, as for a matrix in $M_{n}$, we have $f\left[\varphi^{v}(a)\right]=\varphi^{b}(a)$. Consequently, knowing what is $\varphi^{b}: M_{n} \rightarrow \mathbb{C}$ (cf. Section 3 ) and using the latter equation, we have $f\left[\varphi^{v}(a)\right]=\operatorname{tr}(a b)$. Therefore the standard duality between $\mathbb{M}_{n}$ and $\mathbb{T}_{n}$ gives the estimate $\left|f\left[\varphi^{v}(a)\right]\right| \leq$ $\|b\|_{o}\|a\|_{t}$.

Now, using Hahn/Banach theorem, take $f \in E^{*} ;\|f\|=1$ such that $f\left(\varphi^{v}(a)\right)=\left\|\varphi^{v}(a)\right\|$. Then, by the latter inequality, we have

$$
\left\|\varphi^{v}(a)\right\| \leq\|b\|_{o}\|a\|_{t} .
$$

But it follows from [4, Cor. 3.3] that $f$, being considered as an operator between $E$ and $\mathbb{C}_{\text {min }}$, is completely contractive. Since $\|v\| \leq 1$, this implies that the norm of $f_{n}(v)$ in $M_{n}\left(\mathbb{C}_{\min }\right)=\mathbb{M}_{n}$ is also $\leq 1$, that is $\|b\|_{o} \leq 1$. Therefore the needed estimate for $\left\|\varphi^{v}(a)\right\|$ follows from $(7)$.

Turn to the inverse estimate. By the duality between $\mathbb{M}_{n}$ and $\mathbb{T}_{n}$, there exists $w \in \mathbb{M}_{n} ;\|w\|_{o}=1$ such that $\operatorname{tr}(a w)=\|a\|_{t}$. Set $E:=\mathbb{C}_{\text {min }}$ and consider $w$ in the unit ball of $M_{n}\left(\mathbb{C}_{\text {min }}\right)$. Then $\varphi^{w}: M_{n} \rightarrow \mathbb{C}$ takes $a$ to $\|a\|_{t}$. Therefore, by (2) (with $m=1$ ), we have $\|a\|_{\bullet} \geq\|a\|_{t}$.

Note that the underlying space of the operator space, playing in the smaller category of operator spaces the same role of 'building bricks' for free objects, is again $\mathbb{T}_{n}$ [9, Prop. 2.7]. However, our current object, the space $\widehat{M}_{n}$, is far away to be an operator space. We have already seen this for $n=1$; now we demonstrate this for all $n$.

Take $p \in[1, \infty)$. A matricially normed space $E$ is said to be $p$-convex or $p$-concave if for every matrices $u_{1}, \ldots, u_{n}$ with entries in $E$, we have that $\left.\left\|u_{1} \oplus \ldots \oplus u_{n}\right\| \leq \sum_{k=1}^{n}\left\|u_{k}\right\|^{p}\right)^{\frac{1}{p}}$ or $\left.\left\|u_{1} \oplus \ldots \oplus u_{n}\right\| \geq \sum_{k=1}^{n}\left\|u_{k}\right\|^{p}\right)^{\frac{1}{p}}$, respectively. A space that is $p$-convex and $p$-concave, is called an $L^{p}$-space [4]. Evidently, an operator space is $p$-convex for every $p$.

Proposition 12. The matricially normed space $\widehat{M}_{n}$ is not $p$-convex, in particular, not an $L^{p}$-space, for every $p>1$.

Proof. Suppose the contrary. Take any $q$-concave matricially normed space $E \neq 0$ with $1 \leq q<p$ (for example, $\mathbb{C}_{\max }$ ). Then, according to [15, Theorem 5.3] (cf. also [10, Prop. 3.3]), we have $\mathcal{C C}\left(\widehat{M}_{n}, E\right)=0$. On the other hand, $B_{M_{n}(E)}$ has certainly more than one point. But by virtue of Theorem 2 there is a bijection between the sets $B_{M_{n}(E)}$ and $\mathcal{C C}\left(\widehat{M}_{n}, E\right)$. We came to a contradiction.

Proposition 13. Let $\mathbf{a} \in M_{m}\left(\widehat{M}_{n}\right)$ be a block-diagonal matrix $\mathbf{a}=$ $\left(a_{1} \oplus \ldots \oplus a_{m}\right) ; a_{k} \in M_{n}$. Then $\|\mathbf{a}\|_{m} \geq \frac{1}{n} \sum_{k}\left\|a_{k}\right\|_{t}$. 
Proof. As it is well known, there exist unitary matrices $S_{k}, T_{k}: k=$ $1, \ldots, m$ such that every $S_{k} a_{k} T_{k}$ is a positive diagonal matrix. Note that for $\mathbf{S}:=\left(S_{1} \oplus \ldots \oplus S_{m}\right)$ and $\mathbf{T}:=\left(T_{1} \oplus \ldots \oplus T_{m}\right)$ we have $\mathbf{S a T}=\left(S_{1} a_{1} T_{1} \oplus \ldots \oplus\right.$ $\left.S_{m} a_{m} T_{m}\right)$. Therefore, because of Axiom 2, we can suppose without loss of generality that all $a_{k}$ are positive diagonal matrices.

Set $E:=\mathbb{C}_{\max }$, so we have $M_{m}(E)=\mathbb{T}_{m} ; m \in \mathbb{N}$. Also set $v:=\frac{1}{n} \mathbf{1}$, where 1 is the identity matrix in $\widehat{M}_{n}$, so we have $v \in B_{M_{n}(E)}$. It is easy to see that $\varphi^{v}: M_{n} \rightarrow \mathbb{C}$ takes a to $\operatorname{tr}(v a)=\frac{1}{n} \operatorname{tr}(a)$, hence $\left(\varphi^{v}\right)_{m}(\mathbf{a})$ is the diagonal $m \times m$-matrix with numbers $\frac{1}{n} \operatorname{tr}\left(a_{k}\right)$ on the diagonal. Therefore, by (2), we have $\|\mathbf{a}\| \geq\left\|\left(\varphi^{v}\right)_{m}(\mathbf{a})\right\|_{t}=\frac{1}{n} \sum_{k} \operatorname{tr}\left(a_{k}\right)=\frac{1}{n}\left\|a_{k}\right\|_{t}$.

Note that Proposition 12 could be easily deduced from the previous proposition, without applying to the triviality of the set $\mathcal{C C}\left(\widehat{M}_{n}, E\right)$.

Proposition 13 shows, loosely speaking, that some properties of $\widehat{M}_{n}$ resemble ('up to the multiplier $\frac{1}{n}^{\text {') }}$ ) to those of $L^{1}$-spaces. Nevertheless we have

Proposition 14. The matricially normed space $\widehat{M}_{n} ; n>1$ is not an $L^{1}$-space.

Proof. Suppose the contrary. As a particular case of Proposition 3.2 in [10], every functional $f: E \rightarrow \mathbb{C}_{\max }$, where $E$ is an $L^{1}$-space, contractive in the 'classical' sense, is automatically completely contractive. Since the underlying space of $\widehat{M}_{n}$ is $\mathbb{T}_{n}$, this concerns, in particular, $f: \widehat{M}_{n} \rightarrow$ $\mathbb{C}: a \mapsto \operatorname{tr}(a)$. Consequently, the operator $f_{n}: M_{n}\left(\widehat{M}_{n}\right) \rightarrow M_{n}\left(\mathbb{C}_{\max }\right)$ is contractive. In particular, for $\mathbb{I} \in M_{n}\left(M_{n}\right)$ (see Section 5) we have $\left\|f_{n}(\mathbb{I})\right\|_{t} \leq\|\mathbb{I}\|$. But, by Proposition 7 , we have $\|\mathbb{I}\|=1$, and at the same time $f_{n}(\mathbb{I})=\sum_{i j} \operatorname{tr}\left(e_{i j}\right) e_{j i}$ is the identity matrix in $M_{n}$. Therefore $\left\|f_{n}(\mathbb{I})\right\|=n>1$, a contradiction.

\section{References}

[1] D. P. Blecher, The standard dual of an operator space, Pacific J. Math., No. 1, 153 (1992), 15-30.

[2] D. P. Blecher, C. Le Merdy, Operator Algebras and their Modules, Clarendon Press, Oxford, 2004.

[3] E. G. Effros, Z.-J. Ruan, On matricially normed spaces, Pacific J. Math, 132, (1988) 243-64. 
[4] E. G. Effros, Z.-J. Ruan, Operator Spaces, Clarendon Press, Oxford, 2000 .

[5] A. W. M . Graven, Injective and Projective Banach modules, Indagationes Mathematicae (Proceedings), 82 (1979), 253-272.

[6] A. Grothendieck, Une caracterisation vectorielle-metrique des espaces $L^{1}$, Canadian J. Math., 7 (1955) 552-561.

[7] C. Faith, Algebra: rings, modules and categories, vol. I, SpringerVerlag, Berlin-Heidelberg-New York, 1973

[8] A. Ya. Helemskii, Metric freeness and projectivity for classical and quantum normed modules, Sb. Math, 204 (2013), 1056-1083.

[9] A. Ya. Helemskii, Projectivity for operator modules: approach based on freedom, Rev. Roum. Math. Pures Appl. 59 (2014).

[10] A. Ya. Helemskii, Projective tensor product of proto-quantum spaces, to appear in Colloc. Math., 2017. 05 2017, arXiv:

[11] S. Mac Lane, Categories for the Working Mathematician. SpringerVerlag, Berlin, 1971.

[12] V. I. Paulsen. Completely Bounded Maps and Operator Algebras, Cam. Univ. Press, Cambridge, 2002.

[13] A. Pietsch, History of Banach Spaces and Linear Operators, Birkäuser, Boston-Basel-Berlin, 2007.

[14] J. Pisier, Introduction to Operator Space Theory, Cam. Univ. Press, Cambridge, 2003.

[15] Z.-J. Ruan, Subspaces of $C^{*}$-algebras, J. Funct. Anal., 76 (1988), $217-$ 230 .

Moscow State (Lomonosov) University

Moscow, 111991, Russia

E-mail address: helemskii@rambler.ru 